\title{
Enriched space-time finite elements for fluid-structure interaction
}

\author{
Antoine Legay* — Andreas Zilian** \\ * Laboratoire des Structures et des Systèmes couplés \\ Conservatoire National des Arts et Métiers \\ 2 rue Conté, F-75003 Paris, France \\ antoine.legay@cnam.fr \\ ** Institut für Statik \\ Technische Universität Braunschweig \\ Beethovenstraße 51 \\ 38106 Braunschweig, Allemagne \\ a.zilian@tu-bs.de
}

\begin{abstract}
This paper presents a new numerical approach to deal with fluid structure interaction problems where a thin structure is immersed in an incompressible fluid. Spacetime finite elements are used to discretized the equations using a discontinuous time scheme. In order to take into account the discontinuities due to the structure in the fluid domain, the approximation fluid fields are enriched with appropriate discontinuous functions through a partition of unity (XFEM). The method allows incompatible meshes between fluid and structure, the structure mesh can move freely in the fluid fixed Eulerian mesh.

RÉSUMÉ. Une nouvelle approche pour traiter les problèmes d'interaction fluide structure spécifiques où la structure est mince et immergée dans un fluide incompressible est présentée. Les équations de Navier-Stokes sont discrétisées par des éléments finis espace-temps en utilisant un schéma de Galerkin discontinu en temps. Les différentes discontinuités introduites par la présence de la structure dans le fluide sont modélisées par enrichissement des champs d'approximations de vitesse et de pression par des fonctions appropriées sur le principe de la partition de l'unité (XFEM). Le maillage de la structure peut alors se déplacer librement dans le domaine fluide.

KEYWORDS: fluid structure interaction, space-time finite elements, incompatible meshes, enrichment, partition of unity, XFEM.

MOTS-CLÉS : interaction fluide-structure, éléments finis espace temps, maillages incompatibles, enrichissement, partition de l'unité, XFEM.
\end{abstract}

DOI:10.3166/REMN.17.725-736 @ 2008 Lavoisier, Paris

REMN - 17/2008. Giens 2007, pages 725 to 736 


\section{Introduction}

The modelisation of thin walled structures immersed in fluid flow in computational mechanics is usually based on ALE approaches using moving mesh and/or remeshing strategies. For large structural displacements or rotations these techniques reach certain limitations. Therefore this paper presents a fixed mesh based method utilizing proper enrichment of a space-time finite element approximation (Legay et al., 2006a; Legay et al., 2006b; Kölke et al., 2006; Legay et al., 2007; Zilian et al., 2007). The major conceptual advantage of the space-time finite element method is the straight forward applicability of the enriched finite element technology (XFEM) to describe the evolution of non-smooth solutions within the space-time domain more accurately. The ability and accuracy of the presented method to deal with large body deformations is shown by two applications.

\section{Strong form}

\subsection{Fluid}

The fluid in Eulerian description in the fluid domain $\Omega$ is formulated using the incompressible Navier-Stokes equations:

$$
\begin{aligned}
& \rho v_{i, t}+\rho v_{i, j} v_{j}-\sigma_{i j, j}-g_{i}=0 \text { in } \Omega \\
& v_{i, i}=0 \text { in } \Omega .
\end{aligned}
$$

where $\rho$ is the density, $v_{i}$ is the Eulerian velocity and $g_{i}$ is the gravity force. The stress tensor $\sigma_{i j}$ for the Newtonian fluid is given by

$$
\sigma_{i j}=-p \delta_{i j}+2 \mu e_{i j}
$$

where $p$ is the pressure and $\mu$ is the viscosity. The rate of strain tensor $e_{i j}$ is given by

$$
e_{i j}=\frac{1}{2}\left(v_{i, j}+v_{j, i}\right)
$$

The boundary conditions, in terms of imposed velocity are

$$
v_{i}-v_{i}^{d}=0 \text { on } \partial_{v} \Omega
$$

and in terms of imposed stress state

$$
\sigma_{i j} n_{j}-t_{i}^{d}=0 \text { on } \partial_{t} \Omega
$$

where $v_{i}^{d}$ is the imposed velocity on $\partial_{v} \Omega, t_{i}^{d}$ is the imposed stress vector on $\partial_{t} \Omega$ and $n_{i}$ is the unit outward normal vector to $\partial_{t} \Omega$. 


\subsection{Coupling}

Along the fluid-structure interface $\Gamma$, fluid and structure velocities have to match as well as stress vectors. By noting $v_{i}^{F}$ fluid velocity, $v_{i}^{S}$ structure velocity, $t_{i}^{F}$ fluid stress vector and $t_{i}^{S}$ structure stress vector, the continuity of velocity and stress vector is expressed by

$$
v_{i}^{F}-v_{i}^{S}=0 \quad \text { and } \quad t_{i}^{F}+t_{i}^{S}=0 \text { on } \Gamma .
$$

These conditions are written for a non-slip interface, for a slip interface only the normal parts have to be continuous.

\subsection{Structure}
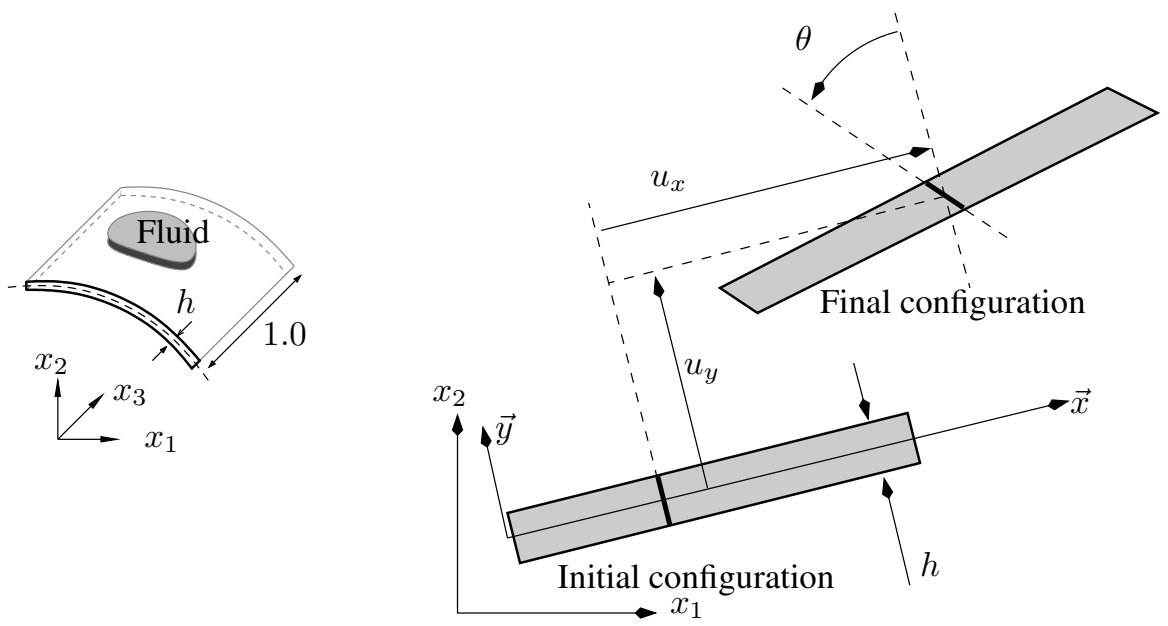

Figure 1. Two-dimensional model of a thin-walled plane structure

The thin structure is modeled as a Timoshenko beam for the two-dimensional examples presented in this paper (Figure 1), while it can be extended to more general three-dimensional structures like membranes or shells. The beam model has to be able to represent large structural motion and displacements, including infinite rigid body rotations. From the kinematics, the Green-Lagrange strain tensor is then written as

$$
\begin{array}{r}
\mathbb{E}=\left[\begin{array}{cc}
e_{1}+y e_{3} & \frac{1}{2} e_{2} \\
\frac{1}{2} e_{2} & 0
\end{array}\right]_{(\vec{x}, \vec{y})} \\
\mathbf{E}=\left(\begin{array}{c}
e_{1} \\
e_{2} \\
e_{3}
\end{array}\right)=\left(\begin{array}{l}
u_{x, x}+\frac{1}{2}\left(u_{x, x}^{2}+u_{y, x}^{2}\right) \\
u_{y, x} \cos \theta-\left(1+u_{x, x}\right) \sin \theta \\
\theta_{, x}\left(\cos \theta+u_{x, x} \cos \theta+u_{y, x} \sin \theta\right)
\end{array}\right)
\end{array}
$$




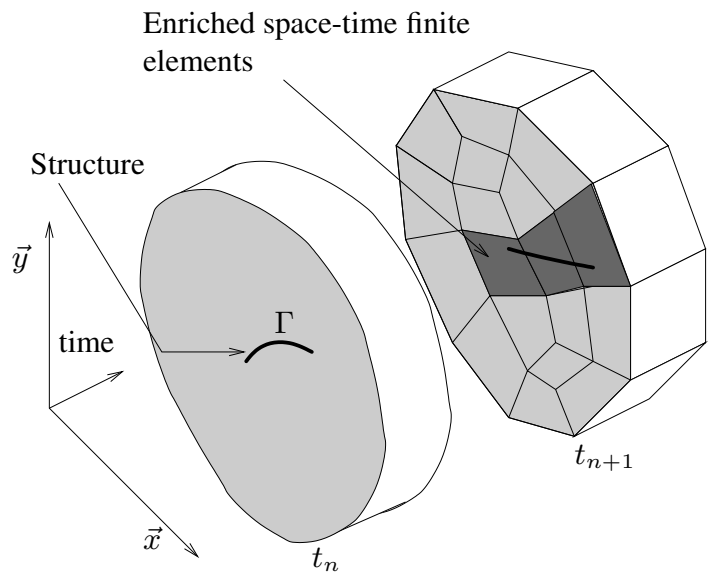

Figure 2. Space-time domain for a thin immersed structure in a fluid flow

while its variation needed in the weak form is

$$
\begin{aligned}
& \delta \mathbf{E}=\left(\begin{array}{lll}
\delta e_{1} & \delta e_{2} & \delta e_{3}
\end{array}\right)^{T} \\
& \delta e_{1}=\delta v_{x, x}+\delta v_{x, x} u_{x, x}+\delta v_{y, x} u_{y, x} \\
& \delta e_{2}=-\delta v_{x, x} \sin \theta+\delta v_{y, x} \cos \theta \\
& -\delta \omega\left(\cos \theta+u_{x, x} \cos \theta+u_{y, x} \sin \theta\right) \\
& \delta e_{3}=\delta v_{x, x} \theta_{, x} \cos \theta+\delta v_{y, x} \theta_{, x} \sin \theta \\
& +\delta \omega_{, x}\left(\cos \theta+u_{x, x} \cos \theta+u_{y, x} \sin \theta\right) \\
& -\delta \omega \theta_{, x}\left(\sin \theta+u_{x, x} \sin \theta-u_{y, x} \cos \theta\right)
\end{aligned}
$$

where $\left(u_{x}, u_{y}\right)$ is the displacement of the corresponding point on the middle line of the beam in the local coordinates system $(\vec{x}, \vec{y}), \theta$ is the rotation of the cross section, $\left(\delta v_{x}, \delta v_{y}\right)$ is the virtual velocity and $\delta \omega$ is the virtual angular velocity.

The generalized force vector $\mathbf{S}$ and the sectional elasticity matrix are introduced such that

$$
\mathbf{S}=\mathbf{C E} \text { with } \mathbf{S}=\left(\begin{array}{c}
N \\
Q \\
M
\end{array}\right) \text { and } \mathbf{C}=\left(\begin{array}{ccc}
E A & 0 & 0 \\
0 & G A & 0 \\
0 & 0 & E I
\end{array}\right)
$$

where $N$ is the normal force, $Q$ is the shear force, $M$ is the bending moment, $E$ is the Young modulus, $G$ is the shear modulus, $A$ is the cross section area and $I$ is the moment of inertia. 


\section{Weak form of the coupled system}

A space-time finite element discretization is used (Figure 2) (Hughes et al., 1988) (Tezduyar et al., 2005). The space-time domain is denoted by $\mathcal{Q}=\Omega \times\left[\begin{array}{ll}0 & t_{f}\end{array}\right]$ where $t_{f}$ is the final time of the study. This space-time domain is cut into slabs from $t_{n}$ to $t_{n+1}$ and solved sequentially for each time slab denoted by $\mathcal{Q}_{n}=\Omega \times\left[\begin{array}{ll}t_{n}^{+} & t_{n+1}^{-}\end{array}\right]$. Initial conditions are given on the boundary of the space-time mesh for $t=0$.

\subsection{Fluid weak form}

The weak form for the fluid domain is written on the space time slab $\mathcal{Q}_{n}$ : Find $\left(v_{i}, p\right)$ such that $\forall\left(\delta v_{i}, \delta p\right)$,

$$
\begin{array}{r}
\int_{\mathcal{Q}_{n}} \delta v_{i} \rho\left(v_{i, t}+v_{i, j} v_{j}\right) \mathrm{d} \mathcal{Q}-\int_{\mathcal{Q}_{n}} \delta v_{i, i} p \mathrm{~d} \mathcal{Q}+\int_{\mathcal{Q}_{n}} \delta v_{i, j} 2 \mu e_{i j} \mathrm{~d} \mathcal{Q} \\
-\int_{\mathcal{Q}_{n}} \delta v_{i} \rho g_{i} \mathrm{~d} \mathcal{Q}-\int_{\partial_{\mathcal{Q}_{n}}} \delta v_{i} t_{i}^{d} \mathrm{~d} S+\int_{\mathcal{Q}_{n}} \delta p v_{i, i} \mathrm{~d} \mathcal{Q} \\
+\int_{\partial \mathcal{Q}\left(t_{n}^{+}\right)} \delta v_{i}\left(t_{n}^{+}\right) \rho\left(v_{i}\left(t_{n}^{+}\right)-v_{i}\left(t_{n}^{-}\right)\right) \mathrm{d} S=0
\end{array}
$$

The last term weakly enforces velocity continuity at time $t_{n}$ from a time slab to an other where $v_{i}\left(t_{n}^{-}\right)$is the known velocity from the previous time slab and $v_{i}\left(t_{n}^{+}\right)$is unknown (discontinuous Galerkin time scheme).

\subsection{Structure weak form}

The weak form for the structure domain is written in the space time domain $\mathcal{Q}_{0}^{s}=$ $\Omega_{0} \times\left[\begin{array}{ll}t_{n} & t_{n+1}\end{array}\right]$ where $\Omega_{0}$ denotes the initial configuration of the structure:

Find $(\mathbf{V}, \mathbf{S})$ such that $\forall(\delta \mathbf{V}, \delta \mathbf{S})$,

$$
\begin{array}{r}
+\int_{\mathcal{Q}_{0}^{s}} \delta \mathbf{V}^{T} \rho \mathbf{G} \dot{\mathbf{V}} \mathrm{d} \mathcal{Q}_{0}+\int_{\mathcal{Q}_{0}^{s}} \delta \mathbf{E}^{T} \mathbf{S} \mathrm{d} \mathcal{Q}_{0}+\int_{\mathcal{Q}_{0}^{s}} \delta \mathbf{S}^{T}\left(\mathbf{C}^{-1} \dot{\mathbf{S}}-\dot{\mathbf{E}}\right) \mathrm{d} \mathcal{Q}_{0} \\
+\int_{\Omega_{0}} \delta \mathbf{V}^{T}\left(t_{n}^{+}\right) \rho\left(\mathbf{V}\left(t_{n}^{+}\right)-\mathbf{V}\left(t_{n}^{-}\right)\right) \mathrm{d} \Omega_{0} \\
+\int_{\Omega_{0}} \delta \mathbf{S}^{T}\left(t_{n}^{+}\right) \mathbf{C}^{-1}\left(\mathbf{S}\left(t_{n}^{+}\right)-\mathbf{S}\left(t_{n}^{-}\right)\right) \mathrm{d} \Omega_{0}=0
\end{array}
$$

where

$$
\mathbf{V}=\left(\begin{array}{c}
v_{x} \\
v_{y} \\
\omega
\end{array}\right) \quad \text { and } \quad \mathbf{G}=\left(\begin{array}{ccc}
A & 0 & 0 \\
0 & A & 0 \\
0 & 0 & I
\end{array}\right)
$$


where $\mathbf{E}$ has the same expression as $\delta \mathbf{E}$ replacing virtual velocities by real ones. The displacement is computed from velocity by time integration. This leads to a mixed formulation for the structure where $\mathbf{S}$ is solved at the structure element level. The two last terms ensure velocity and stress continuity at time $t_{n}$ (discontinuous Galerkin time scheme).

\subsection{Interface weak form}

Velocity continuity on the interface $\Gamma$ is enforced by Lagrange multipliers $\lambda_{i}$ which are additional variables. The following terms are added to the weak form:

$$
\begin{aligned}
\int_{\Gamma \times\left[t_{n} t_{n+1}\right]} \delta \lambda_{i}\left(v_{i}^{F}-v_{i}^{S}\right) \mathrm{d} \Gamma \mathrm{d} t+\int_{\Gamma \times\left[t_{n} t_{n+1}\right]} \delta v_{i}^{F} \lambda_{i} \mathrm{~d} \Gamma \mathrm{d} t \\
-\int_{\Gamma_{0} \times\left[t_{n} t_{n+1}\right]} \delta v_{i}^{S} \frac{\mathrm{d} \Gamma_{t}}{\mathrm{~d} \Gamma_{0}} \lambda_{i} \mathrm{~d} \Gamma \mathrm{d} t
\end{aligned}
$$

The Lagrange multipliers are projected on the reference structural configuration.

\section{Enriched space-time (EST) finite elements for fluid flow}

\subsection{Localization of the structure by level-sets}

The structure is localized in the space-time domain by the zero iso-contour of a level-set $\phi(\underline{x}, t)$ (Sethian, 1999). This function is chosen equal to the signed distance to the interface. Two other orthogonal level-sets $\phi^{1}(\underline{x}, t)$ and $\phi^{2}(\underline{x}, t)$ can be added to defined the two tips of an open structure.

\subsection{First choice of fluid approximation and enrichment}

In this first choice the fluid velocity is approximated in space by quadratic 9 node elements $(Q 2)$ while the pressure is approximated by linear 4 node elements $(Q 1)$. For both velocity and pressure, the time direction is approximated by linear shape functions. This choice satisfied the LBB condition.

The immersed structures involve a pressure discontinuity as well as a velocity gradient discontinuity from one side to the other side of the structure. The pressure approximation is enriched with a Heaviside function (Belytschko et al., 2001):

$$
p(\underline{x}, t)=\sum_{I \in \mathcal{S}} N_{I}^{p}(\underline{x}, t) P_{I}+\sum_{J \in \mathcal{S}^{e n r}} N_{J}^{p}(\underline{x}, t) \operatorname{sign}(\phi(\underline{x}, t)) A_{J}^{p}
$$

where $\mathcal{S}$ is the set of fluid nodes, $N_{I}^{p}(\underline{x}, t)$ is the shape function associated to the pressure node $I, P_{I}$ is the pressure nodal value, $\mathcal{S}^{e n r}$ is the set of fluid enriched nodes, 
and $A_{J}^{p}$ is the new unknown of node $J$. The enriched nodes are the nodes belonging to the elements cut by the interface in the space-time domain (Figure 2).

Using the same notations, the velocity field is enriched by a ramp like function, for instance the absolute value of $\phi(\underline{x}, t)$ :

$$
v_{i}(\underline{x}, t)=\sum_{I \in \mathcal{S}} N_{I}^{v}(\underline{x}, t) V_{I i}+\sum_{J \in \mathcal{S}^{e n r}} N_{J}^{p}(\underline{x}, t)|\phi(\underline{x}, t)| A_{J i}^{v}
$$

\subsection{Alternative choice of fluid fields enrichment}

In this second choice the fluid velocity as well as the pressure are approximated in space by linear 3 node elements $(P 1)$. This choice does not satisfy the LBB condition and a stabilization term is added in the weak form (Masud et al., 1997) (T.E. Tezduyar et al., 1992). Both pressure and velocity are enriched by a Heaviside function:

$$
\begin{aligned}
p(\underline{x}, t) & =\sum_{I \in \mathcal{S}} N_{I}^{p}(\underline{x}, t) P_{I}+\sum_{J \in \mathcal{S}^{e n r}} N_{J}^{p}(\underline{x}, t) \operatorname{sign}(\phi(\underline{x}, t)) A_{J}^{p} \\
v_{i}(\underline{x}, t) & =\sum_{I \in \mathcal{S}} N_{I}^{v}(\underline{x}, t) V_{I i}+\sum_{J \in \mathcal{S}^{e n r}} N_{J}^{p}(\underline{x}, t) \operatorname{sign}(\phi(\underline{x}, t)) A_{J i}^{v}
\end{aligned}
$$

The Heaviside enrichment introduces a strong discontinuity in the velocity field through the interface, the continuity is recover by using two Lagrange multipliers fields $\lambda_{i}^{+}$and $\lambda_{i}^{-}$which enforce continuity from the positive and the negative sides respectively of the interface (in the level-set sence) to the structure. Details of this strategy can be found in reference (Zilian et al., 2007).

\section{Applications}

\subsection{Vibration of a spring supported rigid piston in a fluid channel}

A rigid piston supported by two springs (Figure 3 ) is immersed in a one dimensional fluid channel. The system is initially at rest, a zero pressure $\left(\bar{t}_{1}^{R}=0\right)$ is applied on the right boundary and a constant one is applied at the left boundary $\left(\bar{t}_{1}^{L}=2 \mathrm{~g} / \mathrm{cm} / \mathrm{s}^{2}\right)$ at time $t=0$. The geometrical dimensions of the system are given by $a=1 \mathrm{~cm}, b=8 \mathrm{~cm}, c=2 \mathrm{~cm}$ and $h=0.02 \mathrm{~cm}$. The material parameters are $\rho_{f}=1.25 \cdot 10^{-1} \mathrm{~g} / \mathrm{cm}^{3}, \mu=10^{-2} \mathrm{~g} / \mathrm{cm} / \mathrm{s}, \rho_{s}=5.0 \cdot 10^{-2} \mathrm{~g} / \mathrm{cm}^{3}$, and $k=0.5 \mathrm{~g} / \mathrm{s}^{2}$. The ratio $\xi=m_{f} / m_{s}$ of total fluid mass $m_{f}=\rho_{f} a b$ to total structural mass $m_{s}=\rho_{s}$ ah is $\xi=10^{3}$.

The discontinuous pressure field is presented on Figure 4 for the EST approach using the first enrichment strategy (Section 4.2) as well as for a standard ALE remeshing strategy. The used mesh has ony 8 elements $(8 \times 1)$. The pressure profil is given on Figure 5 and the analytical solution to the problem is exactly recovered. 


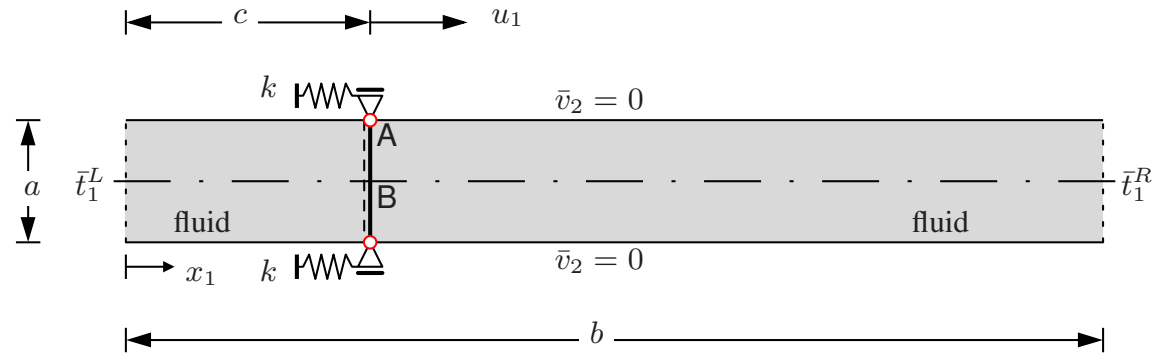

Figure 3. Immersed piston supported by springs in a channel

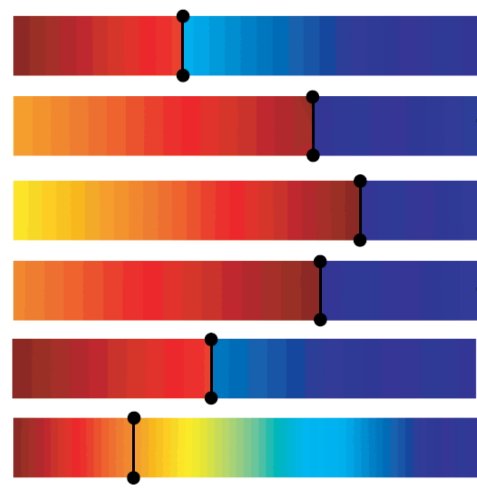

(a) EST approach

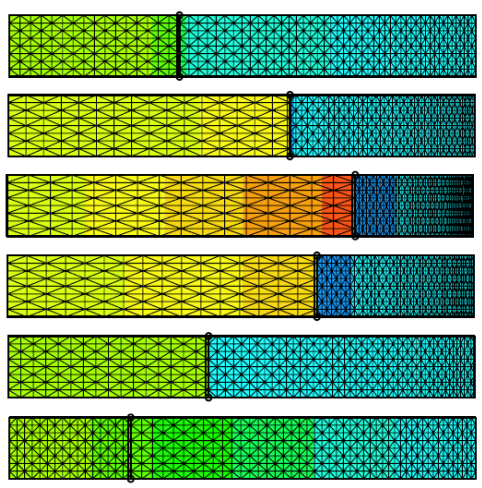

(b) ALE approach

Figure 4. Pressure field for the rigid immersed piston for different time: $1 s, 2 s, 3 s$, $4 s, 5 s$ and $6 s$ (from top)

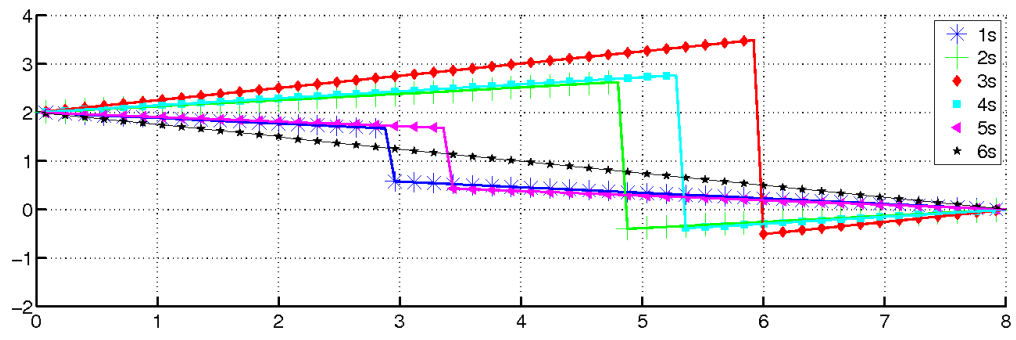

Figure 5. Discontinuous pressure solution $\left(\mathrm{g} / \mathrm{cm} / \mathrm{s}^{2}\right)$ during one period is given in the whole channel 


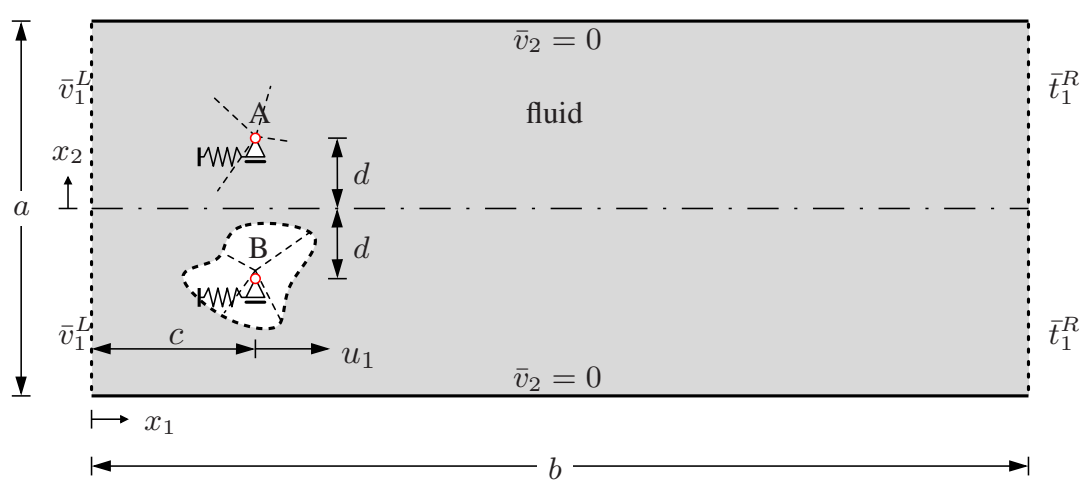

Figure 6. Rotor system: channel flow with immersed flexible and rotating structure (shapes $A$ and $B$ )

\subsection{Two immersed rotating structures in a flow}

Two differently shaped thin-walled structures of width $h$ embedded in a flow channel of dimensions $a=0.2 \mathrm{~cm}$ and $b=2.0 \mathrm{~cm}$ as shown in Figure 6 are considered. In horizontal direction each structure $\left(\rho_{s}=10.0 \mathrm{~g} / \mathrm{cm}^{3}\right)$ is supported by a linear spring of stiffness $k$, and is coupled to the channel flow $\left(\rho_{f}=1.0 \mathrm{~g} / \mathrm{cm}^{3}, \mu_{f}=\right.$ $\left.10^{-3} \mathrm{~g} / \mathrm{cm} / \mathrm{s}\right)$. The enclosed domain inside the solid is filled with fluid $\left(\rho=\rho_{f} / 2\right.$ and $\mu=\mu_{f} / 2$ ). Both structures are free to rotate around their hinges (initial positions given by $c=0.2 \mathrm{~cm}$ and $d=0.05 \mathrm{~cm}$ ) and are fixed in the vertical direction. The stiffeners have no interaction with the enclosed fluid. At the left boundary of the channel the horizontal velocity $\bar{v}_{1}^{L}=1.0 \mathrm{~cm} / \mathrm{s}$ is imposed while on the right the fluid boundary tractions $\bar{t}_{1}^{R}$ are zero $\left(p^{R}=0\right)$.

The curved shapes A and B are generated using the superformula by (Gielis, 2003),

$$
\frac{1}{r}=\left(\left|\frac{1}{\alpha} \cos \left(\frac{\gamma}{4} \theta\right)\right|^{\lambda_{2}}+\left|\frac{1}{\beta} \sin \left(\frac{\gamma}{4} \theta\right)\right|^{\lambda_{3}}\right)^{\frac{1}{\lambda_{1}}},
$$

where $r$ and $\theta$ are polar coordinates, $\alpha, \beta, \gamma, \lambda_{1}, \lambda_{2}$ and $\lambda_{3}$ are real numbers. Table 5.2 lists the used parameters for the rigid $(\mathrm{R})$ and the flexible $(\mathrm{F})$ configurations. The origin of each shape is identical to the hinge point that is connected to the thin-walled structure by stiffeners as shown in Figure 6. Each shape is composed of $n_{P}=120$ nodes and linear thin-walled structure elements.

The narrow flow domain around the immersed shapes is regularly discretized by prismatic space-time finite elements using the second choice for the enrichment (Section 4.3) with equidistant nodes $(\Delta h=0.004 \mathrm{~cm})$. The numerical analysis of the coupled system is performed using a time slab width of $\Delta t=0.001 \mathrm{~s}$ for the time interval $T=[0.0 s, 4.0 \mathrm{~s}]$. 
Table 1. Material and shape parameters (sets $A$ and $B)$ for rigid $(R)$ and flexible $(F)$ rotating structures

\begin{tabular}{|c|c|c|c|c|c|}
\hline setup & $E$ & & $\nu^{\prime}$ & $k$ & $h$ \\
\hline & $\frac{g}{c m s^{2}}$ & & {$[-]$} & {$\left[\frac{g}{s^{2}}\right]$} & {$[\mathrm{cm}]$} \\
\hline $\mathrm{R}$ & - & & - & 1.0 & $1 \cdot 10^{-2}$ \\
\hline F & $10^{4}$ & & $10^{-4}$ & 1.0 & $2 \cdot 10^{-3}$ \\
\hline shape & $\alpha$ & $\beta$ & $\gamma$ & $\lambda_{1}$ & $\lambda_{2}$ \\
\hline $\mathrm{A}$ & 1.0 & 1.0 & 3.0 & 0.1 & 1.8 \\
\hline B & 1.0 & 1.0 & 4.0 & 0.8 & 4.0 \\
\hline
\end{tabular}

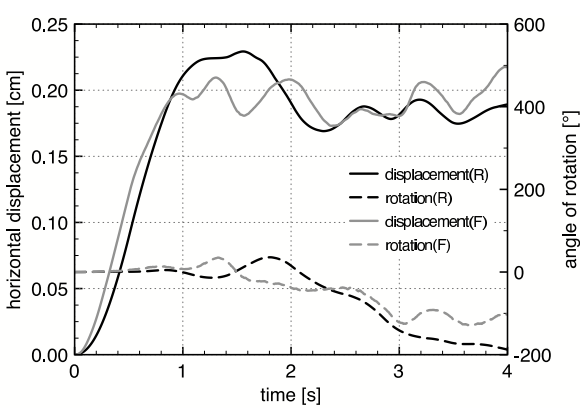

(a)

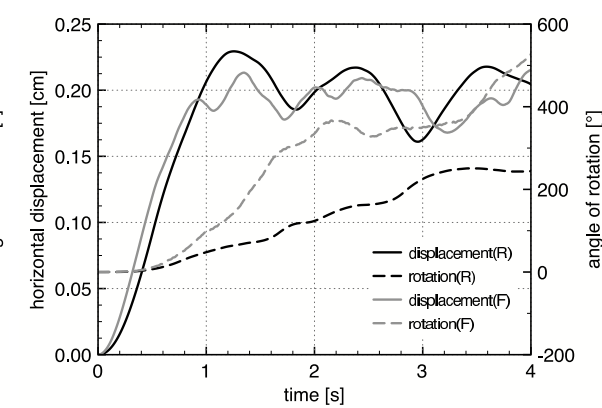

(b)

Figure 7. Horizontal displacement and angle of rotation of immersed rotor shapes $A$ (a) and $B(b)$

In the beginning of the simulation the inflow velocity is linearly increased from zero to the final constant $\bar{v}_{1}^{L}$ within the time interval $T_{v}=[0.0 s, 0.5 s]$. As a consequence the flow field develops $(R e \approx 50)$ and the asymmetric flow situation causes rotational motion of both objects additional to motion in the horizontal direction. Figure 7 shows the solution to horizontal displacements and angle of rotation of the hinges for the rigid and flexible case. The enriched solution fields of pressure and velocity are given in Figure 8 for selected time instants of the configuration involving flexible structures.

This fluid-structure interaction problem can be classified as: instability-induced and movement-induced structural excitation. Initial motion and deformation is caused by the developing fluid instabilities (shear flow) in the typical Kármàn vortex street. Once the flexible structures are set in motion the fluid field changes and a strong coupling of fluid and structure is present. The behavior of the whole system is highly nonlinear due to the motion and deformations of the involved structures in the viscous Navier-Stokes fluid. 

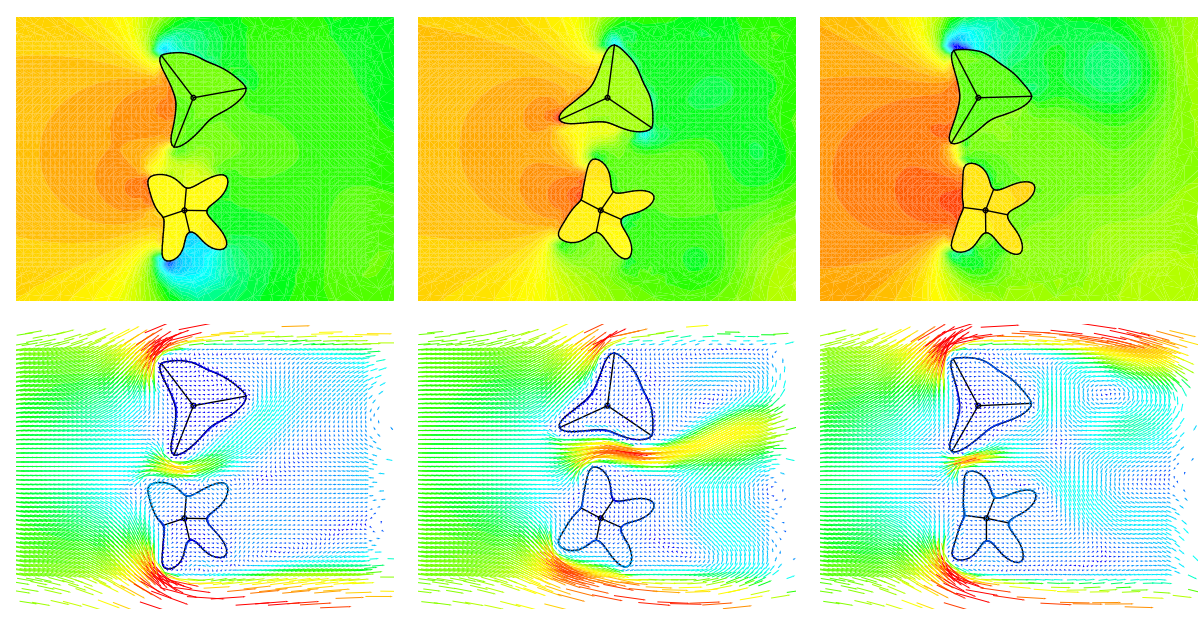

Figure 8. Enriched pressure (top) and velocity (bottom) fields for setup $F$ at times $t=1.0 \mathrm{~s}, t=2.0 \mathrm{~s}$ and $t=3.0 \mathrm{~s}$

The formulation and numerical algorithm of the EST method presented in this paper is capable of handling this nonlinear fluid-structure interaction situation with large structural motion/rotation and deformation.

\section{References}

Belytschko T., Moës T., Usui S., Parimi C., “ Arbitrary Discontinuities in Finite Elements”, International Journal of Numerical Methods in Engineering, vol. 50, n 4, p. 993-1013, 2001.

Gielis J., “ A generic geometric transformation that unifies a wide range of natural and abstract shapes”, American Journal of Botany, vol. 90, n³, p. 333-338, 2003.

Hughes T., Hulbert G., " Space-time finite element methods for elastodynamics: Formulations and error estimates", Computer Methods in Applied Mechanics and Engineering, vol. 66, $\mathrm{n}^{\circ}$ 3, p. 339-363, 1988.

Kölke A., Legay A., “ An enriched space-time finite element method for fluid-structure interaction - Part II: Thin flexible structures", Proceedings of the third European Conference on Computational Mechanics, 5-8 June, 2006.

Legay A., Chessa J., Belytschko T., “ An Eulerian-Lagrangian Method for Fluid-Structure Interaction Based on Level Sets", Computer Methods in Applied Mechanics and Engineering, vol. 195, n 17-18, p. 2070-2087, 2006a.

Legay A., Kölke A., “ An enriched space-time finite element method for fluid-structure interaction - Part I: prescribed structural displacement", Proceedings of the third European Conference on Computational Mechanics, 5-8 June, $2006 \mathrm{~b}$. 
Legay A., Tralli A., “ An Euler-Lagrange enriched finite element approach for fluid-structure interaction”, European Journal of Computational Mechanics, vol. 16, n 2, p. 145-160, 2007.

Masud A., Hughes T., “ A space-time Galerkin/least-squares finite element formulation of the Navier-Stokes equations for moving domain problems", Computer Methods in Applied Mechanics and Engineering, vol. 146, n 1-2, p. 91-126, 1997.

Sethian J., Level Set Methods and Fast Marching Methods Evolving Interfaces in Computational Geometry, Fluid Mechanics, Computer Vision, and Materials Science, Cambridge University Press, 1999.

T.E. Tezduyar M. B., Liou J., “ A new strategy for finite element computations involving moving boundaries and interfaces - The deforming-spatial-domain/space-time procedure: I. The concept and the preliminary numerical tests", Computer Methods in Applied Mechanics and Engineering, vol. 94, n 3, p. 339-351, 1992.

Tezduyar T., Sathe S., Keedy R., Stein K., “S Space-time finite element techniques for computation of fluid-structure interactions", Computer Methods in Applied Mechanics and Engineering, vol. 195, n 17-18, p. 2002-2027, 2005.

Zilian A., Legay A., “ The enriched space-time finite element method (EST) for simultaneous solution of fluid-structure interaction", International Journal of Numerical Methods in Engineering, 2007. 